\title{
Melatonin, an Endogenous Hormone, Modulates Th17 Cells via the Reactive Oxygen Species/TXNIP/HIF-1a Axis to Alleviate Autoimmune Uveitis
}

\section{Dan Liang ( $\sim$ liangdan@gzzoc.com)}

Sun Yat-Sen University Zhongshan Ophthalmic Center Jun Huang

Sun Yat-Sen University Zhongshan Ophthalmic Center Zhuang Li

Sun Yat-Sen University Zhongshan Ophthalmic Center Yunwei Hu

Sun Yat-Sen University Zhongshan Ophthalmic Center

\section{Zuoyi Li}

Sun Yat-Sen University Zhongshan Ophthalmic Center

\section{Yanyan Xie}

Sun Yat-Sen University Zhongshan Ophthalmic Center

\section{Haixiang Huang}

Sun Yat-Sen University Zhongshan Ophthalmic Center

\section{Qian Chen}

Sun Yat-Sen University Zhongshan Ophthalmic Center

\section{Guanyu Chen}

Sun Yat-Sen University Zhongshan Ophthalmic Center

\section{Wenjie Zhu}

Sun Yat-Sen University Zhongshan Ophthalmic Center

\section{Yuxi Chen}

Sun Yat-Sen University Zhongshan Ophthalmic Center

\section{Wenru Su}

Sun Yat-Sen University Zhongshan Ophthalmic Center Xiaoqing Chen

Sun Yat-Sen University Zhongshan Ophthalmic Center

\section{Research}


Keywords: Experimental autoimmune uveitis, Melatonin, Th17/Treg, reactive oxygen species, TXNIP, HIF$1 a$

Posted Date: January 5th, 2022

DOI: https://doi.org/10.21203/rs.3.rs-1192974/v1

License: (c) (1) This work is licensed under a Creative Commons Attribution 4.0 International License. Read Full License 


\section{Abstract \\ Background}

Melatonin, an indoleamine produced by the pineal gland, plays a pivotal role in maintaining circadian rhythm homeostasis. Recently, the strong antioxidant and anti-inflammatory properties of melatonin have attracted attention of researchers. We evaluated the therapeutic efficacy of melatonin in experimental autoimmune uveitis (EAU), which is a representative animal model of human autoimmune uveitis.

\section{Methods}

EAU was induced in mice via immunization with the peptide interphotoreceptor retinoid binding protein 120 (IRBP $1-20)$. melatonin was then administered via intraperitoneal injection to induce protection against EAU. With EAU induction for 14 days, clinical and histopathological scores were employed to evaluate the disease progression. $T$ lymphocytes accumulation, the expression of inflammatory cytokines in the retinas were assessed via flow cytometry and RT-PCR. In vivo and in vitro experiments, T helper 1 (Th1), T helper 17 (Th17) and regulatory $T$ (Treg) cells were detected via flow cytometry, the level reactive oxygen species(ROS) from CD4+ cells were tested via flow cytometry, and the expression of thioredoxininteracting protein (TXNIP) and hypoxia-inducible factor 1 alpha (HIF-1a)proteins were also quantified via western blot analysis, to elucidate the mechanism of melatonin inhibiting EAU.

\section{Results}

Melatonin treatment resulted in notable attenuation of ocular inflammation in EAU mice, evidenced by decreasing optic disc edema, few signs of retinal vasculitis, and minimal retinal and choroidal infiltrates. Mechanistic studies revealed that melatonin restricted the proliferation of peripheral Th1 and Th17 cells and potentiated Treg cells by suppressing their transcription factors. In vitro studies corroborated that melatonin restrains the polarization of retina-specific T cells towards Th17 and Th1 cells in addition to enhancing the proportion of Treg cells. Pretreatment of retina-specific T cells with melatonin failed to induce EAU in naïve recipients. Furthermore, the ROS/ TXNIP/ HIF-1 a pathway was shown to mediate the therapeutic effect of melatonin in EAU.

\section{Conclusions}

Melatonin regulates autoimmune $\mathrm{T}$ cells by restraining effector $\mathrm{T}$ cells and facilitating Treg generation, indicating that melatonin could be a hopeful treatment alternative for autoimmune uveitis.

\section{Background}


Melatonin is an endogenous hormone synthesized mainly by the pineal gland and the retina, and has antioxidant and anti-inflammatory effects $(1-3)$. In autoimmune diseases, melatonin plays additional roles in immune regulation and inhibiting immune responses, in addition to acting as an "immune buffer" (4-7). In a murine experimental model of autoimmune encephalomyelitis (EAE) and in human multiple sclerosis (MS), melatonin was shown to reduce the levels of pro-inflammatory cytokines TNF-a, interleukin (IL)-1 $\beta$, IL-6, and interferon gamma (IFN- $\gamma$ ) under different inflammatory conditions, as well as regulating $T$ helper 17 (Th17) cell differentiation via retinoic acid-related orphan receptor a (RORa) (6). In particular, melatonin reduces the T helper 1 (Th1) response and increases IL-10 production $(8,9)$. However, the therapeutic effects of melatonin in EAU have not yet been elucidated.

Autoimmune uveitis $(\mathrm{AU})$ is a potentially sight threatening intraocular inflammatory process accounting for nearly $10 \%$ of cases of severe visual impairment worldwide, and $25 \%$ of patients of legal blindness in the developing world (10-12). There are many pathological changes in patients with $\mathrm{AU}$, characterized by retinal vasculitis, optic nerve damage, and photoreceptor damage. Current treatment modalities, including biologics and immunosuppresants, are not specific and do not cure the disease. Furthermore, they are often associated with severe adverse effects. Hence, safe and effective alternatives are needed urgently for $\mathrm{AU}$.

EAU is an established animal model of human uveitis caused by interphotoreceptor retinoid binding protein (IRBP) specific effector T cells (Teff) (13). Pathogenic mechanism of AU were undetermined. Studies revealed that increased Teff cells and/or decreased Treg cells mediated initiation and progression of AU (14-16). Thus, suppressing the polarization of Th1 and Th17 cells, and/or potentiating Treg cells are supposed to attenuate $\mathrm{AU}$.

In the current study, we investigated the therapeutic efficacy of melatonin in AU and explored its potential mechanism of action. We demonstrated that systemic administration of melatonin alleviated EAU by decreasing the ratios of Th1 and Th17 effector cells and increasing the ratio of Treg cells, both locally and peripherally. Mechanistic investigations revealed that melatonin restricted the polarization of retinaspecific T cells by downregulating RORyt via the ROS/ TXNIP/HIF-1 a pathway. Our data indicate that melatonin directly regulates autoimmune responses, and holds potential as an alternative therapy option for human AU.

\section{Materials And Methods}

\section{Mice}

C57BL/6 mice (female, 6-8 weeks old; 20-25 g) from the Guangzhou Animal Testing Center (Guangzhou, China) were conducted in specific pathogen-free conditions. The Institutional Animal Care and Use Committee of Zhongshan Ophthalmic Center Sun Yat-sen University approved this study, which was performed in compliance with the ARVO Statement for the Use of Animals in Ophthalmic and Vision Research. 


\section{EAU Induction and treatment with melatonin}

Female mice were subcutaneously immunized with $\mathrm{IRBP}_{1-20}$ (amino acid sequence: GPTHLFQPSLVLDMAKVLLD; GL Biochem, Shanghai, China) emulsified in Freund's Complete Adjuvant (Difco, Detroit, MI, USA) containing Mycobacterium tuberculosis strain H37Ra (Difco; 1:1 v/v), on day 0 and day 2, pertussis toxin (Sigma-Aldrich, St. Louis, MO, USA) was administered intraperitoneally $(17,18)$ .The above methods has also been introduced in detail in our previous study(19). from On the third day of immunization, melatonin (Sigma-Aldrich) was administered intraperitoneally $(10 \mathrm{mg} / \mathrm{kg}$ or $80 \mathrm{mg} / \mathrm{kg}$ in solution that contains $4 \%$ DMSO and $96 \%$ PBS, intraperitoneally). Control group mice were treated with the same volume of vehicle instead of melatonin.

\section{Fundoscopy and histology study in EAU}

On day 14 after immunization, the fundus of the mice was examined by Micron IV Retinal Imaging Microscope (PHOENIX, USA) and scored clinically. Clinical scoring was based on the previous study (18). At day 14 , the mice were euthanized, and eyes were enucleated and stored in $4 \%$ neutral buffered formalin solution for $24 \mathrm{~h}$ at room temperature. Then, samples were dehydrated, embedded and stained. Histopathological changes were evaluated and graded in accordance with previously described criteria (18).

\section{Retina-infiltrating cells isolation}

This method is consistent with our previous published paper(19).

\section{Flow cytometry}

Surface staining of cells with anti-mouse CD4 Percp-Cy5.5 (clone GK 1.5), anti-mouse CD 45 Brilliant Violet 510 (clone 30-F11 ), anti-mouse CD8a PE (clone 53-6.7), anti-mouse CD19 Brilliant Violet 650 (clone 6D5), anti-mouse CD44 antigen-presenting cell (APC) (clone IM7), anti-mouse CD25 PE/Cyanine7 (clone 3C7), anti-mouse CD62L FITC (clone MEL-14), anti-mouse APC/Cyanine7 CD279 (PD-1) (clone 29F.1 A12), anti-mouse CD11c PE (clone N418). Above antibodies are from eBioscience (Carlsbad, CA, USA) or BioLegend (San Diego, CA, USA). For intracellular cytokine IFN-y and IL-17A, the cells were incubated with ionomycin ( $500 \mathrm{ng} / \mathrm{mL})$, PMA $(50 \mathrm{ng} / \mathrm{mL})$, and BFA $(1 \mu \mathrm{g} / \mathrm{mL})$ (Sigma-Aldrich) for $5 \mathrm{~h}$, and after fixation and permeabilization, the cells were stained with anti-mouse IL-17A BV650 (clone TC1118H10.1) and anti-mouse IFN- $y$ BV786 (clone XMG1.2). Transcription factor staining kit was used according to the manufacturer's protocol. For total cellular ROS staining, the cells were incubated with 10 $\mu \mathrm{M} 2^{\prime}, 7^{\prime}$-dichlorodihydrofluorescein diacetate (CAS No. 4091-99-0) for $20 \mathrm{~min}$ at $37^{\circ} \mathrm{C}$ in a cell culture incubator, which were analyzed using a BD LSRFortessa instrument (BD Biosciences, Franklin Lakes, NJ, USA), and the acquired data were processed using FlowJo 10.0 (FlowJo Co., Ashland, OR, USA).

\section{T cell polarization}

Naïve CD4+T cells (CD4+CD62L+CD25-CD44-) were isolated from the lymph nodes and spleen of wild type mice using a commercial kit (Miltenyi Biotec, Gladbach, Germany). The naïve CD4+T cells (purity: 
$95 \% ; 2 \times 10^{5} /$ well) were incubated with anti-CD3/CD28 beads (one bead to five cells) for three days in a 96-well plate. For Treg cell differentiation, the cultures were supplemented with Treg cell differentiation condition (TGF- $\beta 1$ (10 ng/mL; PeproTech, Rocky Hill, NJ, USA) and recombinant human IL-2 (50 U/mL; PeproTech)).

\section{Enzyme-linked immunosorbent assay (ELISA)}

T cells which were isolated from DLNs of EAU mice were preprocessed with melatonin $(200 \mathrm{ng} / \mathrm{mL})$ for 4

$\mathrm{h}$, and stimulated with $10 \mu \mathrm{g} / \mathrm{mL} \mathrm{IRBP}_{1-20}$ for three days. The cell culture supernatants were collected, and the concentrations of IFN- $y$ and IL-17A were assayed using ELISA kits (Invitrogen, Carlsbad, CA, USA). Real-time quantitative polymerase chain reaction (PCR)

Retinas were isolated from the different treatment groups. Total RNA was extracted using TRIzol reagent (Invitrogen) and quantified using a NanoDrop spectrophotometer (ND-1000; NanoDrop Technologies, Wilmington, DE, USA). Total RNA was used to synthesize cDNA using PrimeScript RT Master Mix (Perfect Real Time, TaKaRa Bio Inc., Kusatsu, Japan). Real-time quantitative PCR was performed using SYBR Premix Ex Taq II (TaKaRa Bio Inc.). Glyceraldehyde-3-phosphate dehydrogenase (GAPDH) mRNA was used as an internal control. The relative mRNA expression of IFN-y, IL-17A, and Foxp3 (Sangon Biotech, Shanghai, China) was analyzed using the $2^{-\Delta \Delta C t}$ method.

\section{Western blot assays}

Proteins from cultured cells and DLNs were extracted using whole cell lysis buffer (KeyGen Biotech, Jiangsu, China) and measured the concentration of protein according to the protein assay kit's instructions, which were separated on polyacrylamide-sodium dodecyl sulfate gels, and electrotransferred onto PVDF membrane. Then, blocking with 5\% non-fat dry milk, the PVDF membranes were incubated with TXNIP (rabbit; clone D5F3E; Cell Signaling Technology, Danvers, MA, USA ), HIF-1a (rabbit; clone D1S7W; Cell Signaling Technology) and GAPDH (rabbit; clone D16H11; Cell Signaling Technology) overnight at $4{ }^{\circ} \mathrm{C}$, followed by incubation with the secondary antibody for $2 \mathrm{~h}$. The western blot signals were imaged using enhanced chemiluminescence (Pierce, Rockford, IL, USA). Image J software (NIH, Bethesda, MD, USA) was used to measure the gray scale.

\section{Adoptive transfer assay to induce EAU}

T cells isolated from the DLNs of EAU mice (day 14 after immunization) were stimulated using $\mathrm{IRBP}_{1-20}$ $(10 \mu \mathrm{g} / \mathrm{mL})$ under Th17-polarizing conditions, with or without melatonin $(200 \mathrm{ng} / \mathrm{mL})$ for $72 \mathrm{~h}$, which were washed three times before administration to C57BL/ 6 mice $\left(2 \times 10^{7}\right.$ living cells/mouse) via intraperitoneal injection. The extent of retinal inflammation by fundus and HE was evaluated on day 14 .

\section{IRBP-specific responses}

In vitro study, the cells $\left(4 \times 10^{5}\right)$ from the DLNs of EAU mice were cultured in 96-well plates with IRBP $_{1-20}$ under Th1-polarizing conditions (ImmunoCult 10953; STEMCELL Technologies, Vancouver, Canada) or

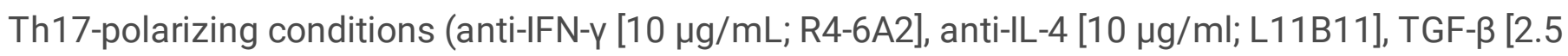


$\mathrm{ng} / \mathrm{mL}], \mathrm{IL}-6[25 \mathrm{ng} / \mathrm{mL}]$, and IL-23 [10 ng/mL]) in the presence or absence of melatonin $(0,2,20$, or $200 \mathrm{ng} / \mathrm{mL}$ ). After three days, the cells were harvested, and flow cytometry was used for the intracellular inflammatory cytokine.

\section{Statistical analysis}

Data were presented as means \pm standard deviation(SD). The Student's $t$-test, Mann-Whitney test, or oneway analysis of variance was selected according to the data sets normality. All data were analyzed by GraphPad Prism 8.2 (GraphPad Software, Inc., La Jolla, CA, USA). P $<0.05$ was considered to be statistically significant.

\section{Results}

\section{Melatonin treatment protected mice from retinal injury after EAU induction}

Melatonin was administered daily from the third day post-immunization to determine the therapeutic effects of melatonin on EAU. An intraperitoneal dose of 10 or $80 \mathrm{mg} / \mathrm{kg}$ was chosen based on a preliminary study. Systemic toxicity (evaluated as mortality rate, behavior, alterations in weight, appearance) were not observed with the chosen dose of melatonin. Fundus of EAU mice treated with vehicle revealed severe chorioretinal lesions, infiltrations, vasculitis and prominent retinal folds. Mice treated by melatonin ( 10 and $80 \mathrm{mg} / \mathrm{kg} /$ day) exhibited less vasculitis, and fewer infiltrated inflammatory lesions (Fig. 1A). Histopathological analyses revealed scattered inflammatory cell infiltrations and extensive retinal folding with detachment in the vehicle group. Intraperitoneal administration of melatonin (10 and $80 \mathrm{mg} / \mathrm{kg}$ ) considerably decreased retinal inflammatory cells infiltration and folds (Fig. 1C). Melatonin treatment significantly decreased the clinical scores and histopathological scores of mice with EAU compared with the vehicle group (Fig. 1B, D). Further, $80 \mathrm{mg} / \mathrm{kg}$ melatonin treatment showed better therapeutic effect and was chosen for subsequent in vivo studies. This observation indicated that melatonin significantly reduced the severity of EAU.

\section{Melatonin treatment alters intraocular Teff/Treg immune balance}

Considering that the active lymphocytes infiltrate into the eye is a critical step in retinal inflammation and pathological injury in $\mathrm{AU}$, assays were performed to judge whether melatonin could reduce $\mathrm{T}$ lymphocytes aggregates in the eye. Intraocular cells were isolated, stained, and analyzed by flow cytometry 14 days after immunization. Melatonin treatment greatly lessened intraocular CD4+T cell accumulation compared with treatment with vehicle only (Fig. 2A).

Dysregulated Teff cells and/or Treg cells contribute to the initiation and progression of autoimmune diseases. Therefore, we analyzed the intraocular production of IFN-y and IL-17A by Th1 and Th17 cells, 
respectively, to evaluate the effect of melatonin treatment on the CD4+T cell immune responses. Melatonin treatment significantly decreased the frequency of Th1 and Th17 cells (Fig. 2B, C). Interestingly, melatonin treatment significantly increased the proportion of $\mathrm{CD} 4+F$ oxp3+Treg cells (Fig. 2D). Subsequently, we investigated the gene expression of IL-17A, IFN- $y$, and Foxp3 in the retina using real-time PCR. The results revealed that melatonin markedly suppressed the gene expression of IL17A and IFN- $\gamma$, while increasing the gene expression of Foxp3. Overall, melatonin ameliorated retinal inflammation by downregulating intraocular Th17/Th1 cells and upregulating Treg cells.

\section{Melatonin regulated Th1/Th17 and Treg cell balance in peripheral lymphoid organs of EAU mice}

It is supposed that IRBP-specific T cells are constituted in the DLNs and spleen and migrate through the impaired blood retinal barrier to the retina tissues, resulting in intraocular inflammation and retinal structural destruction. Hence, we evaluated the effects of melatonin on Teff and Treg cells in DLNs and spleen. Single cells were prepared from DLNs and spleen and analyzed by flow cytometry. Melatonin decreased the frequency of Th1 and Th17 cells compared with the vehicle group (Fig. 3A, B). Regulatory $T$ cells were investigated because they are known to suppress inflammation by modulating Teff cells (20). Melatonin markedly increased the percentage of CD25+FoxP3+ cells in the DLNs and spleens of EAU mice (Fig. 3C). The effects of melatonin on Th1 and Th17 cells were further explored by analyzing their respective transcription factors, T-bet and RORyt. Compared with the vehicle group, melatonin treatment dramatically decreased the levels of T-bet and RORyt (Figs. 3D, E). These data suggested that melatonin treatment alters the balance of effector $T$ cells and regulatory $T$ cells in the periphery, contributing to the inhibition of retinal inflammatory injury.

\section{Melatonin inhibits IRBP $1-20$-specific Th cell responses and promotes Treg cell differentiation in vitro}

To clarify the mechanism(s) by which melatonin regulates Th1 and Th17 cells, we investigated the effects of melatonin on Th1 and Th17 cell differentiation in vitro. Cells were obtained from the DLNs of EAU mice and stimulated with IRBP ${ }_{1-20}$ under Th1- or Th17-polarizing conditions, with or without melatonin. The presence of melatonin in the culture system $(0,2,20$, and $200 \mathrm{ng} / \mathrm{mL})$ inhibited the generation of IFN- $\gamma$ - or IL-17A-CD4+ T cells in a dose-dependent manner (Fig. 4A, B). Furthermore, supernatants of the culture systems were collected and assayed for IL-17A and IFN- $\gamma$ using ELISA. Compared to the blank control, cells stimulated by $\mathrm{IRBP}_{1-20}$ produced markedly higher levels of IL-17A and IFN-y. However, the addition of melatonin mitigated IL-17A and IFN-y production significantly (Fig. 4C, D). Since T-bet and RORYt are transcription factor for Th1 and Th17, we also examined the effect of melatonin on T-bet and RORyt. Our results demonstrated that melatonin suppressed T-bet and RORyt in vitro. (Fig. 4E, F). In addition, naïve CD4+T cells were sorted and polarized under Treg conditions in the presence of melatonin or not. We found melatonin facilitated Treg cell differentiation dramatically 
(Fig. 4G). Together, these results support the notion that melatonin restricts IRBP ${ }_{1-20}$-specific $T$ cell responses and promotes Treg cell differentiation.

To further demonstrate the inhibitory ability of melatonin on the pathogenicity of IRBP $_{\text {I- } 20}$-specific $T$ cells, we carried out the adoptive transfer experiment. The DLNs of EAU mice were isolated to make single cells suspension, which was stimulated by $\operatorname{IRBP}_{1-20}(10 \mathrm{ug} / \mathrm{ml})$, separately, with a vehicle, and melatonin (200 $\mathrm{ng} / \mathrm{mL}$ ) under Th17-polarizing conditions for three days. Equal numbers of $\operatorname{IRBP}_{1-20}$-specific $T$ cells pretreated with different treatment were adoptively transferred to the C57BL/6J mice via the tail vein. Two weeks later, we found melatonin treatment attenuated the disease severity (Fig. 4H). At the end of the experiment, the retinas histological analysis verified the clinical manifestation (Fig. 4I). These outcomes indicate that melatonin regulated the effector functions of Th17 cells to alleviate EAU.

\section{Melatonin attenuates uveitis mainly by regulating the activation and functional status of CD4 $+\mathrm{T}$ cells}

Since EAU is a T cell-mediated CNS- Autoimmune disease, we investigated whether melatonin could influence $\mathrm{CD} 4+\mathrm{T}$ cell activation and function, and found that the expression of the $T$ cell inhibitory receptor, programmed cell death protein 1 (PD-1), was significantly increased after melatonin treatment (Fig. 5A). To further investigate whether melatonin treatment inhibited lymphocyte activation, we analyzed the expression of the early activation marker CD69 on cells from spleen and DLN. Melatonin treatment significantly inhibited CD69+ cells in DLN, but not in the spleen (Fig. 5B). Subsequently, we analyzed naïve CD4+ CD62+CD44- T cells (Th0) that were not activated by antigen during EAU. Interestingly, the percentages of naive T cells in the spleen and DLNs were much higher in the melatonintreated group than in the vehicle-treated group (Fig. 5C).

As part of the innate immune system, dendritic cells (DCs) are the first to recognize and process antigens and present them to $T$ cells to initiate autoimmune diseases. To ascertain whether melatonin treatment modulated DCs, we analyzed cells from DLNs and spleen of melatonin-treated EAU mice by flow cytometry. The frequency of DCs showed little difference between the two groups (Fig. 5D). Furthermore, flow cytometry results revealed negligible effects of melatonin on the frequency of CD4+, CD8, and CD19+ cells (Fig. 5E, F). These data suggested that the therapeutic effect of melatonin in EAU is mediated mainly by the activation of CD4+T cells.

\section{Melatonin suppresses Th17 cells from EAU mice via the oxidative stress/TXNIP/HIF-1a axis}

Reactive oxygen species (ROS) are produced by enzymatic/nonenzymatic metabolic redox reactions, which can lead to various diseases and disorders, such as uveitis(21). In particular, the redox protein thioredoxin (TRX) can produce free radicals, while TXNIP is an endogenous inhibitor of TRX. The TRXTXNIP interaction plays an critical role in redox regulation and participates in cell proliferation and growth. Increased levels of ROS can disrupt the TRX-TXNIP homeostasis (22-24). Previously research reported that downstream HIF-1a signaling could facilitate Th17 differentiation $(25,26)$; so we 
investigated whether melatonin alleviated uveitis via the oxidative stress/TXNIP/HIF-1a signaling axis. We found that the levels of ROS in CD4+T cells in the lymph nodes and spleen were significantly reduced in the melatonin treatment group (Fig. 6A). Further in vitro experiments showed increased ROS after CD4+T activation and melatonin inhibited ROS as well as CD4+T activation (Fig. 6B). EAU mice exhibited increased levels of TXNIP and HIF-1a. Meanwhile, melatonin treatment greatly reduced TXNIP and HIF-1a levels (Fig. 6C). To verify the in vivo findings, we carried out in vitro experiments to determine whether melatonin could suppress ROS/TXNIP/HIF-1a and Th17 differentiation. DLN cells isolated from EAU mice were cultured with $\mathrm{IRBP}_{1-20}$ in the presence of melatonin or not under Th17-polarizing conditions for 72 h. ROS levels in CD4+T cells were significantly reduced by melatonin in a dose-dependent manner (Fig. 6D). Furthermore the expression of TXNIP and HIF-1 a proteins were induced by IRBP ${ }_{1-20}$ stimulation, and attenuated by melatonin in a dose-dependent manner (Fig. 6E). Together, Above data supported the notion that melatonin suppresses Th17 cells from EAU mice via the ROS/TXNIP/ HIF-1a signaling axis.

\section{Discussion}

In the present study, we probed into the beneficial effects of melatonin in EAU, a characteristic model of human non-infectious uveitis. Systemic administration of melatonin to mice inhibited the infiltration of inflammatory cells and facilitated Treg cells into the eyes. Melatonin could suppress the differentiation and function of uveitogenic effectors and potentiate regulatory $T$ cells, which are crucial participants in the initiation and progression of uveitis. Additional mechanistic studies revealed that melatonin can effectively control disease progression by inhibiting the ROS/TXNIP/HIF-1a signaling axis.

Melatonin is produced mainly by the pineal gland and exerts multiple biological activities, including antioxidation, anti-aging, and immunomodulatory properties, besides its circadian effect (1-3). Melatonin has been proven to exert beneficial effects as an immune modulator in multiple autoimmune diseases, such as MS $(6,8)$ and inflammatory bowel disease (IBD) (27)'(28). However, the role of melatonin in patients with RA and in animal models of collagen-induced arthritis (CIA) remains controversial. Some studies suggest that melatonin has adverse effects in patients with RA and may increase the disease severity in the CIA model (29-31), while other studies have documented the anti-inflammatory and immunoregulatory properties of melatonin. Tang et al. reported that melatonin mitigates the disease severity of rheumatoid arthritis by attenuating TNF- $\alpha$ and IL-1 $\beta$ expression in synovial fibroblasts and reducing cartilage degradation (32). Korkmaz also advocated that melatonin is an appropriate adjunctive therapy for RA (33). However, whether or not melatonin can effectively attenuate disease severity in patients with AU remains undetermined. Herein, we conducted experiments on mice and found that melatonin alleviated the severity of EAU.

Th17 cells have been reported to conduce to the pathogenesis of autoimmune diseases, such as MS, rheumatoid arthritis, IBD, and psoriasis, and are potential targets for immunotherapy (34). Th17 also plays a major pathogenic role in uveitis (35-37). Researchers have shown that melatonin can influence the differentiation of Th17 and decrease Th17 infiltration in EAE models $(5,7)$. Furthermore, melatonin treatment increased the percentage of Treg cells in the blood of patients with systemic lupus 
erythematosus and in EAE model $(6-8,38)$. In addition, our results also found that melatonin can suppress auto-reactive Th17 cells and Th1 cells, but facilitate Treg cells both in vitro and in vivo.

As a potential candidate for the therapy of inflammatory diseases, whether melatonin has an effect on immune cells are a major concern. We evaluated the peripheral DC, T cell, and B cell populations of EAU mice treated with melatonin. Melatonin had no effects on the frequency of DCs in the spleen and DLNs. Furthermore, no obvious decrease was observed in the frequency of CD4+T cells, CD8+T cells, or CD19+ $B$ cells after in melatonin-treated mice. Mauricio et al. confirmed that melatonin acts directly on CD4+T cells rather than controlling them indirectly via DCs (6). Considering that EAU is a T cell-mediated disorder, and T cell activation is the first step in initiating downstream events, we analyzed the effect of melatonin on CD4+T activation, and found that the expression of both CD69 and CD44 was inhibited by melatonin. Alvarez-Sanchez $\mathrm{N}$ et al. reported that melatonin-treated mice significantly reduce the expression of CD44(8). However, the underlying mechanism remains to be elucidated.

ROS acts as a second messenger in T cell receptor signal transduction, which is essential for cell activation and effector functions $(39,40)$. Previous studies have shown that biological stimuli induce cells to produce a large amount of ROS, and the increased levels of ROS disrupts the intracellular TRXTXNIP balance (41). TXNIP is a pivotal endogenous negative regulator of cellular redox balance $(22,42$, 43) that is highly expressed in immune cells and plays a role in regulating lymphocyte cycle progression and proliferation in the immune system $(41,44)$. Of note, Previte DM et al. reported that increased ROS in $\mathrm{CD} 4+\mathrm{T}$ cells was associated with $\mathrm{CD} 4+\mathrm{T}$ cell activation (45). In our study, we also verified that melatonin reduced the levels of ROS in CD4+T cells, as well as TXNIP, accompanied with the activation of CD4+T cells. However, the effects of ROS/TXNIP redox metabolism on CD4+T cell function and the mechanism of Teff / Treg immune homeostasis are not clearly revealed. Considering above, we further explored the HIF-1a expression during the process, which was reported to be greatly affected by ROS (46) and promoted Th17 differentiation through direct transcriptional activation of RORyt $(25,47-49)$. As expected, our results shown that melatonin significantly inhibited the increase in the levels of ROS and TXNIP expression in EAU mice, in addition to inhibiting the expression of HIF-1a. These were consistent with various studies that HIF-1a, a crucial metabolic sensor, regulated the balance between Treg and Th17 cell differentiation and had a strong interaction with ROS/TXNIP, the down-regulation of which

resulted in alleviation on EAU. Moreover, Schraml et al. also demonstrated that mice with HIF-1 $\mathrm{a}^{-/-} \mathrm{T}$ cells are resistant to $E A E$ induction (50). Therefore, our results contribute to the available literature by demonstrating the effect of melatonin on cellular immune balance from the perspective of the redox balance of CD4+T cells.

\section{Conclusion}

Taken together, our study demonstrates the therapeutic effect of melatonin on EAU for the first time. Melatonin significantly reduced local and systemic inflammatory responses and modulated T cell populations through the ROS/TXNIP/HIF-1 a signal axis. These results not only enrich our understanding 
of the mechanism of EAU, but also expand the clinical application potential of melatonin and offer a new and compelling option for the treatment of $\mathrm{AU}$.

\section{Abbreviations}

AU

Autoimmune uveitis

EAU

experimental autoimmune uveitis

ELISA

Enzyme-linked immunosorbent assay

HIF-1a

hypoxia-inducible factor 1 alpha

IL-1 $\beta$

interleukin (IL)-1 $\beta$

IFN- $\mathrm{Y}$

interferon gamma

$\operatorname{IRBP}_{1-20}$

interphotoreceptor retinoid binding protein 1-20

PD-1

programmed cell death protein 1

ROS

reactive oxygen species

RT-PCR

Real-time quantitative polymerase chain reaction

Th1

T helper 1

Th17

T helper 17

Treg regulatory $T$ cells

Teff effector T cells

TXNIP

thioredoxin-interacting protein

\section{Declarations}

Ethics approval and consent to participate 
The Institutional Animal Care and Use Committee of Zhongshan Ophthalmic Center Sun Yat-sen University approved this study.

\section{Consent for publication}

Not applicable

\section{Availability of data and materials}

This article included all relevant data about this study.

\section{Competing interests}

The authors declare no competing interests.

\section{Funding}

This work was supported by the National Natural Science Foundation of China (No. 81870649); Guangzhou Science and Technology Plan Project (NO. 202102010208).

\section{Authors' contributions}

$\mathrm{JH}$ and $\mathrm{ZL}$ designed the study, created model, analyzed data, and writed manuscript. YX, GC and QC were helpful in manuscript writing. $\mathrm{YH}, \mathrm{ZL}$ and $\mathrm{YC}$ provided experiments guide and supervised this study. $\mathrm{HH}$, WZ and WS helped in revision of the manuscript. XC and DL were responsible for conception and design, revision of the manuscript, and final manuscript approval. All authors approved the submitted version for publication.

\section{Acknowledgments}

Not applicable

\section{References}

1. Legros C, Chesneau D, Boutin JA, Barc C, Malpaux B. Melatonin from cerebrospinal fluid but not from blood reaches sheep cerebral tissues under physiological conditions. J Neuroendocrinol. 2014;26(3):151-63.

2. Reiter RJ, Tan DX, Kim SJ, Cruz MH. Delivery of pineal melatonin to the brain and SCN: role of canaliculi, cerebrospinal fluid, tanycytes and Virchow-Robin perivascular spaces. Brain Struct Funct. 2014;219(6):1873-87.

3. Stehle JH, Saade A, Rawashdeh O, Ackermann K, Jilg A, Sebestény T, et al. A survey of molecular details in the human pineal gland in the light of phylogeny, structure, function and chronobiological diseases. Journal of pineal research. 2011;51(1):17-43. 
4. Carrillo-Vico A, Guerrero JM, Lardone PJ, Reiter RJ. A review of the multiple actions of melatonin on the immune system. Endocrine. 2005;27(2):189-200.

5. Ren W, Liu G, Chen S, Yin J, Wang J, Tan B, et al. Melatonin signaling in T cells: Functions and applications. Journal of pineal research. 2017;62(3).

6. Farez MF, Mascanfroni ID, Mendez-Huergo SP, Yeste A, Murugaiyan G, Garo LP, et al. Melatonin Contributes to the Seasonality of Multiple Sclerosis Relapses. Cell. 2015;162(6):1338-52.

7. Ma F, Hao H, Gao X, Cai Y, Zhou J, Liang P, et al. Melatonin ameliorates necrotizing enterocolitis by preventing Th17/Treg imbalance through activation of the AMPK/SIRT1 pathway. Theranostics. 2020;10(17):7730-46.

8. Alvarez-Sanchez N, Cruz-Chamorro I, Lopez-Gonzalez A, Utrilla JC, Fernandez-Santos JM, MartinezLopez A, et al. Melatonin controls experimental autoimmune encephalomyelitis by altering the $T$ effector/regulatory balance. Brain Behav Immun. 2015;50:101-14.

9. Chen SJ, Huang SH, Chen JW, Wang KC, Yang YR, Liu PF, et al. Melatonin enhances interleukin-10 expression and suppresses chemotaxis to inhibit inflammation in situ and reduce the severity of experimental autoimmune encephalomyelitis. Int Immunopharmacol. 2016;31:169-77.

10. Miserocchi E, Fogliato G, Modorati G, Bandello F. Review on the worldwide epidemiology of uveitis. Eur J Ophthalmol. 2013;23(5):705-17.

11. Caspi RR. A look at autoimmunity and inflammation in the eye. The Journal of clinical investigation. 2010;120(9):3073-83.

12. Muñoz-Fernández S, Martín-Mola E. Uveitis. Best Pract Res Clin Rheumatol. 2006;20(3):487-505.

13. Gery I, Caspi RR. Tolerance Induction in Relation to the Eye. Frontiers in immunology. 2018;9:2304.

14. Lyu C, Bing SJ, Wandu WS, Xu B, Shi G, Hinshaw SJ, et al. TMP778, a selective inhibitor of RORyt, suppresses experimental autoimmune uveitis development, but affects both Th17 and Th1 cell populations. Eur J Immunol. 2018;48(11):1810-6.

15. Su W, Chen X, Zhu W, Yu J, Li W, Li Y, et al. The cAMP-Adenosine Feedback Loop Maintains the Suppressive Function of Regulatory T Cells. Journal of immunology (Baltimore, Md : 1950). 2019;203(6):1436-46.

16. Silver PB, Horai R, Chen J, Jittayasothorn Y, Chan CC, Villasmil R, et al. Retina-specific T regulatory cells bring about resolution and maintain remission of autoimmune uveitis. Journal of immunology (Baltimore, Md : 1950). 2015;194(7):3011-9.

17. Li Z, Chen X, Chen Y, Li H, Yu J, Li Y, et al. Teriflunomide suppresses T helper cells and dendritic cells to alleviate experimental autoimmune uveitis. Biochemical pharmacology. 2019;170:113645.

18. Agarwal RK, Silver PB, Caspi RR. Rodent models of experimental autoimmune uveitis. Methods in molecular biology (Clifton, NJ). 2012;900:443-69.

19. Huang J, Li Z, Hu Y, Chen G, Li Z, Xie Y, et al. Azithromycin modulates Teff/Treg balance in retinal inflammation via the mTOR signaling pathway. Biochemical pharmacology. 2021;193:114793. 
20. Sakaguchi S, Sakaguchi N, Shimizu J, Yamazaki S, Sakihama T, Itoh M, et al. Immunologic tolerance maintained by $\mathrm{CD} 25+\mathrm{CD} 4+$ regulatory $\mathrm{T}$ cells: their common role in controlling autoimmunity, tumor immunity, and transplantation tolerance. Immunol Rev. 2001;182:18-32.

21. Hsu SM, Yang CH, Teng YT, Tsai HY, Lin CY, Lin CJ, et al. Suppression of the Reactive Oxygen Response Alleviates Experimental Autoimmune Uveitis in Mice. International journal of molecular sciences. 2020;21(9).

22. Lu J, Holmgren A. The thioredoxin antioxidant system. Free Radic Biol Med. 2014;66:75-87.

23. Tang L, Zhang $C$, Yang $Q$, Xie H, Liu D, Tian H, et al. Melatonin maintains inner blood-retinal barrier via inhibition of p38/TXNIP/NF-KB pathway in diabetic retinopathy. J Cell Physiol. 2021;236(8):584864.

24. Liu Q, Zuo R, Wang K, Nong FF, Fu YJ, Huang SW, et al. Oroxindin inhibits macrophage NLRP3 inflammasome activation in DSS-induced ulcerative colitis in mice via suppressing TXNIP-dependent NF-kB pathway. Acta Pharmacol Sin. 2020;41(6):771-81.

25. Dang EV, Barbi J, Yang HY, Jinasena D, Yu H, Zheng Y, et al. Control of $T(H) 17 / T($ reg) balance by hypoxia-inducible factor 1. Cell. 2011;146(5):772-84.

26. Shi LZ, Wang R, Huang G, Vogel P, Neale G, Green DR, et al. HIF1alpha-dependent glycolytic pathway orchestrates a metabolic checkpoint for the differentiation of TH17 and Treg cells. The Journal of experimental medicine. 2011;208(7):1367-76.

27. Pentney PT, Bubenik GA. Melatonin reduces the severity of dextran-induced colitis in mice. Journal of pineal research. 1995;19(1):31-9.

28. Chojnacki C, Wisniewska-Jarosinska M, Walecka-Kapica E, Klupinska G, Jaworek J, Chojnacki J. Evaluation of melatonin effectiveness in the adjuvant treatment of ulcerative colitis. Journal of physiology and pharmacology : an official journal of the Polish Physiological Society. 2011;62(3):327-34.

29. El-Awady HM, El-Wakkad AS, Saleh MT, Muhammad SI, Ghaniema EM. Serum melatonin in juvenile rheumatoid arthritis: correlation with disease activity. Pak J Biol Sci. 2007;10(9):1471-6.

30. Sulli A, Maestroni GJ, Villaggio B, Hertens E, Craviotto C, Pizzorni C, et al. Melatonin serum levels in rheumatoid arthritis. Ann N Y Acad Sci. 2002;966:276-83.

31. Hansson I, Holmdahl R, Mattsson R. Constant darkness enhances autoimmunity to type II collagen and exaggerates development of collagen-induced arthritis in DBA/1 mice. Journal of neuroimmunology. 1990;27(1):79-84.

32. Huang CC, Chiou CH, Liu SC, Hu SL, Su CM, Tsai CH, et al. Melatonin attenuates TNF-a and IL-1 $\beta$ expression in synovial fibroblasts and diminishes cartilage degradation: Implications for the treatment of rheumatoid arthritis. Journal of pineal research. 2019;66(3):e12560.

33. Korkmaz A. Melatonin as an adjuvant therapy in patients with rheumatoid arthritis. British journal of clinical pharmacology. 2008;66(2):316-7.

34. Yasuda K, Takeuchi Y, Hirota K. The pathogenicity of Th17 cells in autoimmune diseases. Semin Immunopathol. 2019;41(3):283-97. 
35. Bing SJ, Shemesh I, Chong WP, Horai R, Jittayasothorn Y, Silver PB, et al. AS101 ameliorates experimental autoimmune uveitis by regulating Th1 and Th17 responses and inducing Treg cells. Journal of autoimmunity. 2019;100:52-61.

36. Napier RJ, Lee EJ, Davey MP, Vance EE, Furtado JM, Snow PE, et al. T cell-intrinsic role for Nod2 in protection against Th17-mediated uveitis. Nat Commun. 2020;11(1):5406.

37. Chen X, Su W, Wan T, Yu J, Zhu W, Tang F, et al. Sodium butyrate regulates Th17/Treg cell balance to ameliorate uveitis via the Nrf2/HO-1 pathway. Biochemical pharmacology. 2017;142:111-9.

38. Medrano-Campillo P, Sarmiento-Soto H, Álvarez-Sánchez N, Álvarez-Ríos Al, Guerrero JM, RodríguezPrieto I, et al. Evaluation of the immunomodulatory effect of melatonin on the T-cell response in peripheral blood from systemic lupus erythematosus patients. Journal of pineal research. 2015;58(2):219-26.

39. Yang Y, Bazhin AV, Werner J, Karakhanova S. Reactive oxygen species in the immune system. International reviews of immunology. 2013;32(3):249-70.

40. Sena LA, Li S, Jairaman A, Prakriya M, Ezponda T, Hildeman DA, et al. Mitochondria are required for antigen-specific $T$ cell activation through reactive oxygen species signaling. Immunity. 2013;38(2):225-36.

41. Muri J, Heer S, Matsushita M, Pohlmeier L, Tortola L, Fuhrer T, et al. The thioredoxin-1 system is essential for fueling DNA synthesis during T-cell metabolic reprogramming and proliferation. Nat Commun. 2018;9(1):1851.

42. Park MJ, Kim DI, Lim SK, Choi JH, Kim JC, Yoon KC, et al. Thioredoxin-interacting protein mediates hepatic lipogenesis and inflammation via PRMT1 and PGC-1a regulation in vitro and in vivo. $J$ Hepatol. 2014;61(5):1151-7.

43. Hwang J, Suh HW, Jeon YH, Hwang E, Nguyen LT, Yeom J, et al. The structural basis for the negative regulation of thioredoxin by thioredoxin-interacting protein. Nat Commun. 2014;5:2958.

44. Muri J, Thut $H$, Kopf $M$. The thioredoxin-1 inhibitor Txnip restrains effector T-cell and germinal center B-cell expansion. Eur J Immunol. 2021;51(1):115-24.

45. Previte DM, Piganelli JD. Reactive Oxygen Species and Their Implications on CD4(+) T Cells in Type 1 Diabetes. Antioxid Redox Signal. 2018;29(14):1399-414.

46. Pouysségur J, Mechta-Grigoriou F. Redox regulation of the hypoxia-inducible factor. Biol Chem. 2006;387(10-11):1337-46.

47. Sakaguchi S, Yamaguchi T, Nomura T, Ono M. Regulatory T cells and immune tolerance. Cell. 2008;133(5):775-87.

48. Zheng SG, Gray JD, Ohtsuka K, Yamagiwa S, Horwitz DA. Generation ex vivo of TGF-beta-producing regulatory T cells from CD4+CD25- precursors. Journal of immunology (Baltimore, Md : 1950). 2002;169(8):4183-9.

49. Zheng SG, Wang JH, Gray JD, Soucier H, Horwitz DA. Natural and induced CD4+CD25+ cells educate CD4+CD25- cells to develop suppressive activity: the role of IL-2, TGF-beta, and IL-10. Journal of immunology (Baltimore, Md: 1950). 2004;172(9):5213-21. 
50. Schraml BU, Hildner K, Ise W, Lee WL, Smith WA, Solomon B, et al. The AP-1 transcription factor Batf controls $T(H) 17$ differentiation. Nature. 2009;460(7253):405-9.

\section{Figures}

\section{Fig. 1}
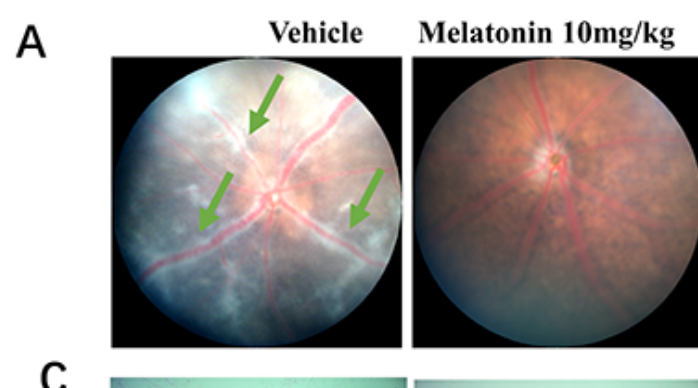

C

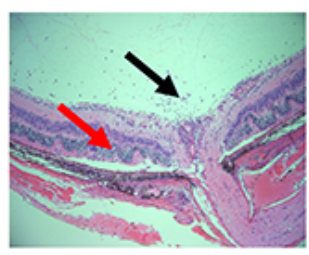

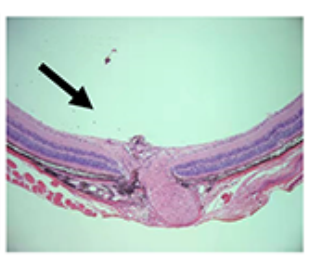
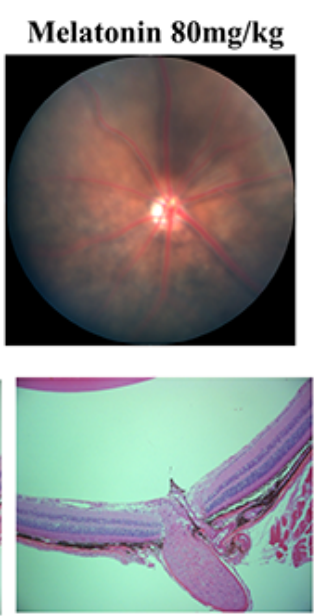

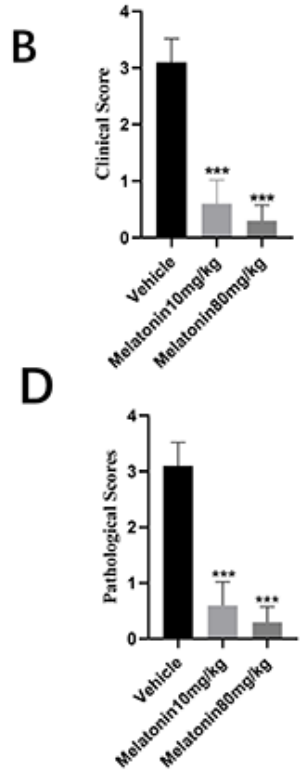

\section{Figure 1}

Melatonin treatment protected mice from retinal injury after EAU induction. C57BL/6J mice immunized with $\operatorname{IRBP}_{1-20}$ were treated with melatonin at different dosages $(0,10$, or $80 \mathrm{mg} / \mathrm{kg} /$ day) daily from day three after immunization. (A) Representative pictures of fundoscopic examination in the vehicle group and melatonin (10,80 mg/kg/day) group at fourteen days after immunization (green arrow: vasculitis). (B) The clinical scores of EAU were performed 14 days after immunization $(n=5)$. (C) Representative pictures of histopathologic examination in the vehicle group and melatonin (10 or $80 \mathrm{mg} / \mathrm{kg} / \mathrm{day}$ ) group (black arrow: inflammatory cells, red arrow: retinal folding with detachments). (D) The pathological scores of EAU via HE were performed 14 days after immunization $(n=5)$. Representative data from three independent experiments. ${ }^{\star \star \star} \mathrm{P}<0.001$ vs. vehicle group.

\section{Figure 2}

Melatonin treatment alters intraocular Teff/Treg immune balance. (A) Flow cytometric analysis of eyeinfiltrating cells revealed greatly decreased levels of CD4+T cells after melatonin treatment. (B-D) Flow cytometric analysis of intracellular expression of interferon gamma (IFN- $\mathrm{y}$ ) and interleukin (IL)-17 by 
CD4+T cells harvested from the eyes of experimental autoimmune uveitis mice on day 14 of treatment with the vehicle (dimethyl sulfoxide) or melatonin $(n=3)$. $(E-G)$ The expression levels of IL-17A, IFN- - , and Foxp3 mRNA in the retinas were measured using real-time polymerase chain reaction 14 days after immunization. Melatonin treatment downregulated the expression of IL-17A and IFN- $\gamma$ mRNA, and upregulated the expression of Foxp3 mRNA $(n=4)$. The representative values from three independent experiments. ${ }^{*} \mathrm{P}<0.05,{ }^{\star} * \mathrm{P}<0.01,{ }^{\star * *} \mathrm{P}<0.001$

\section{Figure 3}

Melatonin regulated Th1/Th17 and Treg cell balance in peripheral lymphoid organs of EAU mice. (A-C) The levels of CD4+IFN- $\gamma+$, CD4+IL-17A+, and CD25+Foxp3+ T cells in the DLNs and spleens of EAU mice were evaluated by flow cytometry fourteen days after immunization. Melatonin treatment decreased the levels of T helper 1 (Th1) and Thelper 17 (Th17) cells and increased the levels of Treg cells significantly $(n=5)$. (D-E) The lineage transcription factors of Th1 and Th17 were also analyzed by flow cytometry 14 days after immunization. CD4+T-bet+ and CD4+RORyt+T cells in the DLNs and spleens of EAU mice ( $\mathrm{n}=$ 4) were obviously inhibited by melatonin treatment. The representative data from three independent experiments. ${ }^{*} \mathrm{P}<0.01,{ }^{* \star *} \mathrm{P}<0.001$.
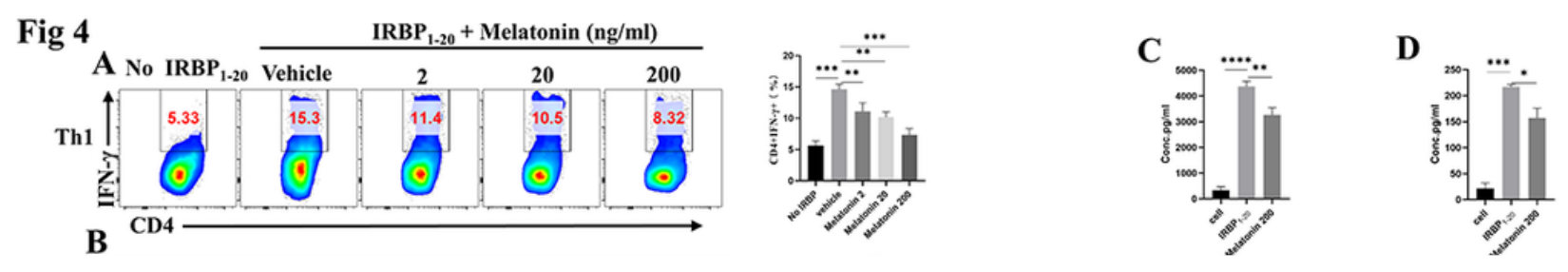

\section{Figure 4}


Melatonin inhibits IRBP $_{1-20}$-specific Th cell responses and promotes Treg cell differentiation in vitro. (AB) Cells from DLNs of EAU mice were stimulated with $10 \mu \mathrm{g} / \mathrm{mL} \operatorname{IRBP}_{1-20}$ under T helper 1 (Th1) or T helper 17 (Th17)-polarizing conditions with vehicle (dimethyl sulfoxide) or different concentrations of melatonin $(0,2,20$, or $200 \mathrm{ng} / \mathrm{mL})$ for $72 \mathrm{~h}$. After polarization towards Th1 or Th17 cells, the CD 4+ cell population was assessed for the expression of interferon (IFN)-y or interleukin (IL)-17A expression, respectively, by flow cytometry $(n=4)$. (C-D) The levels of IL-17A and IFN- $\gamma$ in supernatants were measured via enzyme-linked immunosorbent assay (ELISA). (E-F) The transcription factors of polarized Th1 and Th17 cells (T-bet and RORyt, respectively) were assayed by flow cytometry; melatonin treatment decreased both in a dose dependent manner $(n=4)$. $(G)$ Naïve CD4+T cells were polarized to Treg cells with or without melatonin for three days. CD25+Foxp3+ cell expression was analyzed by flow cytometry $(\mathrm{n}=4)$. $(\mathrm{H}-\mathrm{I})$ Cells were isolated from DLNs of EAU mice and stimulated with $\mathrm{IRBP}_{1-20}$ peptide under Th17-polarizing conditions in the absence (vehicle) or presence of melatonin $(200 \mathrm{ng} / \mathrm{mL})$. After three days, viable cells were collected and adoptively transferred to C57BL/ 6 wild-type mice (2 million cells/mouse; $n=4)$. (H) Disease was observed by fundoscopy at day 14 (green arrow: vasculitis). (I) Histology scores and representative pictures of hematoxylin and eosin-stained histology slides on day 14 (black arrow: inflammatory cells, red arrow: retinal folding with detachments). The representative data from three independent experiments. ${ }^{\star} P<0.05,{ }^{\star} \mathrm{P}<0.01,{ }^{\star \star \star} \mathrm{P}<0.001, \star \star \star \star \mathrm{P}<0.0001$.

\section{Figure 5}

\section{Melatonin attenuates uveitis mainly by regulating the activation and functional status of CD4 +T cells}

(A-C) Frequencies of CD4+CD69+, CD4+PD-1+ T cells, and CD44-CD62+ naïve CD4+ T cells from the spleen and DLNs of immunized mice in two groups. (D) Frequencies of CD11C+ cells from immunized mice in the vehicle-treated and melatonin-treated groups were measured by flow cytometry. (E-F) Frequencies of CD19 (B cells), CD8, and CD4+T cells in immunized mice were measured by flow cytometry ( $n=4$; four animals per group in three independently performed experiments). ${ }^{n s} P>0.05, * P<$ $0.05, * * \mathrm{P}<0.01, * \star * \mathrm{P}<0.001$.

\section{Figure 6}

Melatonin suppresses Th17 cells from EAU mice via the oxidative stress/TXNIP/HIF-1a axis (A) The levels of reactive oxygen species (ROS) were analyzed by flow cytometry. (B) Effects of different concentrations of melatonin on ROS levels during early activation of naive CD4+T cells. (C) Western blot analyses TXNIP and HIF-1a in the CD4+T cells from DLNs of EAU mice. (D) DLN cells from EAU mice were stimulated for $72 \mathrm{~h}$ with $10 \mu \mathrm{g} / \mathrm{mL}$ IRBP $_{1-20}$ under Th17-polarizing conditions in the absence (vehicle) or presence of melatonin $(0,2,20$, or $200 \mathrm{ng} / \mathrm{mL})$. CD $4+T$ cells were assessed for ROS 
expression by flow cytometry. (E) DLN cells from EAU mice were stimulated for $72 \mathrm{~h}$ with $10 \mu \mathrm{g} / \mathrm{mL}$ $\mathrm{IRBP}_{1-20}$ under Th17-polarizing conditions in the absence (vehicle) or presence of melatonin $(0,2,20,200$ $\mathrm{ng} / \mathrm{ml}$ ). Th17-polarized DLN cells was assessed for TXNIP and HIF-1a expression via western blotting, respectively. The representative data from three independent experiments. ${ }^{\star} P<0.05$, ${ }^{\star *} P<0.01$, ${ }^{\star \star \star} P<$ 0.001 . 